\title{
Paul Wouters, Anne Beaulieu, Andrea Scharnhorst, \& Sally Wyatt (eds) (2013) Virtual Knowledge: Experimenting in the Humanities and the Social Sciences. Cambridge, MA: The MIT Press.
}

\author{
Dominique Vinck \\ dominique.vinck@unil.ch
}

Virtual Knowledge addresses the new ways in which knowledge is produced in the humanities and social sciences (HSS) with the advent of digital technology. Refusing to limit knowledge to its cognitive dimension alone, Paul Wouters, Anne Beaulieu, Andrea Scharnhorst and Sally Wyatt, together with their colleagues from the Virtual Knowledge Studio for the HSS at the KNAW (Koninklijke Nederlandse Akademie van Wetenschappen), deal with knowledge as a set of practices linked to actors, instruments and institutions. They question what has changed with the use of digital tools: acquisition, representation and circulation of knowledge; knowledge itself; and the relations between researchers, disciplines, deciders and other emerging actors. They position these changes in relation to several global developments: growth of the science system and its opening up to various types of public; new forms of governance requiring researchers to report on their activities and their results; and the institution of big science as a model for all disciplines.

The hypothesis put forward is that technological change offers the ideal opportunity to analyse these transformations because it encourages the actors concerned to think about their practices, research topics and scientific productions. Researchers are thus urged to explain and formalise their practices and are exposed to epistemic culture shocks caused by the interdisciplinary coalitions they build. Thinking is fuelled by promises, disappointments, controversies and revised promises.
The authors have opted to structure their analyses around the notion of Virtual Knowledge rather than e-science (associated too much with the ideas of quantification and data-oriented research), cyber science (referring to infrastructure questions) or e-research (focusing on research practices), in order to concentrate on academic knowledge (which, in the field of Literature, does not just concern scientific research) and non-academic knowledge. As for the notion of virtual (rather than digital), it is used to underline a distance in relation to technology and refers to the potential and dynamics at work with respect to knowledge production conditions.

The book, above all, targets researchers in the humanities and social sciences and, indirectly, the institutions supporting and supervising the development of these disciplines. It comprises seven chapters exploring various themes: shifts in authority and expertise, emotional labour in scientific collaboration, uncertainty in relation to tools and models, the rhetoric of visualisation tools, massive data processing, broader access to data, and research promises and agendas.

All the authors focus on what is new in terms of knowledge production and on the effects of the transformations on actors, practices and contingencies. Several cases are analysed and compared in each chapter. Each author also takes particular care with the conceptual dimension of their analysis framework.

On the other hand, the authors of each chapter use different approaches to address the themes 
covered. Anne Beaulieu, Sarah de Rijke and Bas van Heur apply a neo-institutionalist approach and examine pre-existing sociotechnical networks to explain the tensions between reproduction and change in relations of authority and the legitimate distribution of expertise among actors. They study the database of an ethnographic museum, the sharing of street art photos on Flickr and a municipal site dealing with local cultural heritage. In their chapter on emotional labour in scientific collaborations, Smiljana Antonijević, Stefan Dormans and Sally Wyatt probe into ordinary practices, especially the work of articulation, persuasion and the care taken in interpersonal relations (sociology of invisible work in health by Susan L. Star and in the (SCW), and their effects on digital resources. They take the case of an international collaboration in economic and social history as well as their own experience writing this book. Matthijs Kouw, Charles van den Heuvel and Andrea Scharnhorst examine Paul Otlet's universal knowledge classification system, Buckminster Fuller's World Game simulation and Paul Forester's mathematical model of world balance. They explore different forms of uncertainty, underlining their creative potential and homing in on the advantage offered by the HSS, which are used to dealing with ambiguous data, uncertain relations and multiple interpretations. Having chosen to focus on visualisation tools (geographic information systems), Rebecca Moody, Matthijs Kouw and Victor Bekkers explore their rhetorical aspect and study the transformations to power relations (approach stemming from government studies) between experts, citizens and policy makers in relation to the ability of the tools to include different actors and the way access to data is shared. Their case studies concern the management of water and flooding, epidemics in livestock farming and fine particles in the air. Clement Levallois, Stephanie Steinmetz and Paul Wouters address big data as they explore the fate of sociological survey methods faced with flows of data from the daily use of digital communication networks together with the fate of decision-making models and theories in economics with the rise of neuroimaging data. The authors question how both sociology and economics (their knowledge questions, topics, practices and statements) respond to and/or are affected by such data. Hence, they report on the disciplines' empirical research traditions, put the claims for radical change linked to dataintensive research into perspective and study the reaction of researchers and their community. They underline the challenges linked to authority and the processes according to which novelty is assimilated. Clifford Tatum and Nicholas Jankowski study open access to data, conceptualising the notion of opening in terms of inclusiveness and transparency. They draw on Wanda Orlikowski's idea of the dual nature of technology, as both agency and structure, in order to examine changes in the formal and informal communication practices of researchers: publication of books and reviews, e-mails, blogs, and enriched publications. Finally, Jan Kok and Paul Wouters turn to the sociology of promises, narrative analysis and the study of research agendas in order to study the series of promises-demands in family history and populations, since the creation of large databases springing up from population registers through to the emergence of visualisation tools and the analysis of social networks.

All of these contributions are of great interest but it would have been interesting to cross-apply the different approaches to the different themes, for example by examining what institutions and pre-existing sociotechnical relations do to the emotional labour of building and maintaining digital collaborations or, conversely, by looking at how the work of attending to relations influences the distribution of scientific authority.

The book as a whole nevertheless constitutes an asset to research in these areas with its rich offering of multiple cases and its broad variety of themes and conceptual approaches. The result is not a cross-referenced thesis but rather a plurality of lines of attack questioning the extent of changes versus continuities, socio-epistemic reconfigurations, and the embedding of novelty in the pre-existing sociotechnical fabric. The book goes much further than simply deconstructing the claims of novelty and paradigmatic change. It reports on the temporality of the transformations at work, their ever partial reality, epistemic and institutional changes, and the challenges of legitimacy and authority. 